\title{
Blastocystis genetic diversity among children of low-income daycare center in Southeastern Brazil
}

\author{
Ana Paula Oliveira-Arbex ${ }^{\mathrm{a}}$, Érica Boarato David ${ }^{\mathrm{a}, \mathrm{b}}$, Semíramis Guimarães ${ }^{\mathrm{a}, *}$

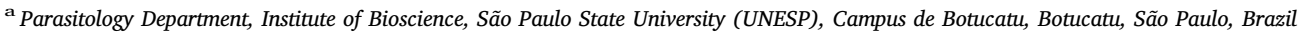 \\ ${ }^{\mathrm{b}}$ Sagrado Coração University (USC), Department of Health Sciences, Bauru, São Paulo, Brazil
}

\section{A R T I C L E I N F O}

\section{Keywords:}

Blastocystis

Children

Daycare center

Molecular epidemiology

Subtypes

\begin{abstract}
A B S T R A C T
Blastocystis, an unicellular anaerobic eukaryote, is known to be a very common intestinal parasite found in humans and animals fecal samples worldwide. Currently, there is an increasing interest to yield insights into its prevalence and diversity in human populations living in poor and deprived areas. In this study, we describe the prevalence and genetic variability of Blastocystis isolates obtained from daycare center attendees aged 0 to 6 years and staff, as well as some children family members and their dogs in a low-income community in São Paulo State, Brazil. A total of 181 stool samples (123 from daycare children, 14 from workers, 44 from household members and 20 from dogs) were submitted to DNA extraction, tested by polymerase chain reaction (PCR) targeting the SSUrDNA gene and the amplicons retrieved were sequenced. The prevalence of Blastocystis was $40.7 \%(50 / 123)$ in children, $28.6 \%(4 / 14)$ in workers and 50\% (22/44) in household members. No dog was found positive. Of the 76 PCR products generated, 57 were successfully sequenced. Four subtypes were identified and the most common were ST1 (54.4\%) and ST3 (33.3\%), followed by ST2 (7.0\%) and ST7 (5.3\%). The intrasubtype analysis revealed a total of 10 different alleles previously reported. No statistically significant correlation was observed between subtypes and sociodemographic variables analyzed. Here, the following findings must be highlighted: (1) predominance of subtypes 1 and 3, a pattern that has been observed in many populations worldwide; (2) absence of ST4, a common subtype in Europe but rarely detected in South America's human populations and, (3) human infection with ST7, a subtype primarily found in birds but occasionally seen in human infections, raising the possibility of zoonotic transmission.
\end{abstract}

\section{Introduction}

The enteric protist Blastocystis, this enteric protist has been increasingly isolated in human population as well as in a wide range of hosts including non-human mammals, birds, reptilians, amphibians and, to a lesser extent, insects (Yoshikawa et al., 2016a). This ubiquitous parasite has a worldwide distribution and it is one of the most common intestinal protists found in parasitological surveys carried out in both developed and developing countries. Although widespread, its real geographic distribution is not well known and high prevalence rates have been reported in economically disadvantaged areas where inadequate access to safe water and sanitation services ensures conditions for the parasite transmission (Alfellani et al., 2013a; Tan, 2008).

Despite its early discovery, Blastocystis has come into focus only recently and a number of outstanding issues regarding epidemiology and pathogenicity still need investigation (Clark et al., 2013). Clinical relevance of Blastocystis infection is controversial because this parasite has been found in healthy individuals as well as in patients with gastrointestinal disorders. Interestingly, over the last two decades, studies have assembled evidence of a possible role of Blastocystis in irritable bowel syndrome (Clark et al., 2013; Poirier et al., 2012).

On the other hand, considerable progress has been made towards understanding its genetic diversity and host specificity. Based on gene analysis of the small subunit ribosomal RNA (SSUrDNA), Blastocystis genus exhibits a marked genetic diversity and it is known to comprise at least 17 distinct ribosomal lineages identified among mammals and birds isolates, the so-called subtypes (Alfellani et al., 2013b; Stensvold, 2013a). To date, nine different Blastocystis subtypes (ST1-ST9) have been found in human infections with a predominance of STs 1-4 which can also infect non-human primates, other mammals and even birds (Stensvold and Clark, 2016). Among these human subtypes, ST9 is so far found in humans while STs 5-8 are more frequent in hosts as hoofed animals (ST5), birds (ST6 and ST7) and in non-human primates (ST8) (Stensvold and Clark, 2016). Also, STs 10-17 have only been reported

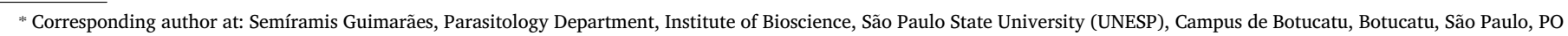
Box: 510; CEP: 18618-970, Brazil.

E-mail address: sgviana@ibb.unesp.br (S. Guimarães). 
in animals.

Notwithstanding the progress made over the last two decades, current interest has focused on the distribution and relevance of Blastocystis subtypes in populations living in low-income areas. Those include Asia (Belleza et al., 2015; Nithyamathi et al., 2016; Pandey et al., 2015; Sanpool et al., 2017), Africa (El Safadi et al., 2014; Poulsen et al., 2016), the Middle East (AbuOdeh et al., 2016; Dogan et al., 2017) and also in Latin America (David et al., 2015; Macchioni et al., 2016; Malheiros et al., 2011; Melo et al., 2017; Ramirez et al., 2014, 2016, 2017; Sánchez et al., 2017; Villegas-Gómez et al., 2016). Recently, the limited data from South America was highlighted (Ramirez et al., 2016, 2017). To date, few investigations were performed in Colombia (Ramirez et al., 2014, 2016, 2017; Sánchez et al., 2017), to a lesser extend in Bolivia (Macchioni et al., 2016) and Argentina (Casero et al., 2015). Particularly in Brazil, there have been only three publications of diversity in Blastocystis. One focused on members of an Amazon indigenous community (Malheiros et al., 2011), while the two others conducted among fishing village dwellers (David et al., 2015) and patients attending a university hospital (Melo et al., 2017).

Despite current advances, there is still the expectation for further insights into Blastocystis epidemiology, especially in endemic foci where conditions ensure the parasite spreading putting the population at risk of infection. The aim of the current study was to evaluate the prevalence and the genetic diversity of Blastocystis isolates related to asymptomatic infection in children attending a day-care center in a small low-income community. In addition, Blastocystis infection was also assessed in daycare staff and some household members, as well dogs in close contact with children.

\section{Materials and methods}

\subsection{Study population and fecal samples collection}

Sample collection was carried out in a day-care center placed in a low-income community of Botucatu town, São Paulo State, Brazil $\left(22^{\circ} 52^{\prime} 20^{\prime \prime} \mathrm{S}, 48^{\circ} 26^{\prime} 38^{\prime \prime} \mathrm{W}\right)$. Fecal specimens, sociodemographic data (age, sex, parents'/guardians' education level, household income, water source and sanitation infrastructure) and genomic DNA were obtained through a previous epidemiological survey to assess the occurrence and the frequency of Giardia genotypes infecting children and staff members of a daycare center, as well as household members and their dogs (Oliveira-Arbex et al., 2016). A total of 181 fecal samples were assessed, 123 of which were from daycare children, 14 from center employees, 44 from household members and 20 from dogs. All the protocols were previously approved by the Research Ethics Committees of the Botucatu Medical School, UNESP (protocol number 3898/2011 CEP) and Animal Experimentation/Biosciences Institute/UNESP (protocol number 306 CEEA).

\subsection{DNA extraction, PCR and sequence analysis}

Genomic DNA previously extracted from stool samples using the QIAamp $^{\circledR}$ Stool mini kit (Qiagen, Hilden, Germany) were subjected to PCR and, the amplification of a $600 \mathrm{bp}$ fragment of the Blastocystis
SSUrDNA was carried out according to standard protocol (Scicluna et al., 2006). For determining the nucleotide sequences, PCR products were purified by the QIAquick PCR purification kit (Qiagen, Hilden, Germany) and sequenced on both strands by the Sequencing Service Macrogen, Inc. (Seoul, Korea). Nucleotide sequences generated were aligned with each other and reference sequences (ST1-ST9) downloaded from GenBank using Clustal X (Larkin et al., 2007). Blastocystis subtypes were identified by BLAST searches (http://blast.ncbi.nlm.nih. gov/Blast.cgi) and the alleles were identified at the Blastocystis database (http://pubmlst.org/blastocystis). Phylogenetic analysis were performed with MEGA software v. 7.0 (www.megasoftware.net) and a phylogram was constructed using Neighbor-joining algorithm. Bootstrap analysis was applied to evaluate the reliability of clusters by using 1000 replicates and values of $<50 \%$ were not shown. The nucleotide sequences obtained in the present study were deposited on GenBank under the accession numbers KY801207 to KY801263.

\subsection{Statistical analysis}

Data were entered into the Epi Info software v. 7.0 (Centers for Disease Control and Prevention, USA). Chi-square $\left(\chi^{2}\right)$ test was applied to verify associations between Blastocystis subtypes and some sociodemographic and environmental factors such as age, sex, family size and income, hygiene habits, water supply, source of drinking water, sewage availability, latrine system, type of housing and contact with domestic and companion animals (the level of significance was $P<0.05)$. Blastocystis subtypes distribution between daycare children, workers and household members were tested for significance by the adjusted Wald test $(P<0.0001)$ using the statistical software SAS 9.2 (SAS Institute, Cary, NC, USA).

\section{Results}

All 181 human fecal samples were screened for Blastocystis by PCR and 76 of them (41.9\%) were identified as positive. None of the dog specimens generated PCR products for Blastocystis. The frequency of human infection was $40.7 \%(50 / 123)$ in children, $28.6 \%(4 / 14)$ in workers and $50 \%(22 / 44)$ in household members. At the time of this survey, Blastocystis-infected and non-infected individuals reported none gastrointestinal complaints.

Of those 76 PCR products, 57 (38 isolates from children, 4 from workers and 15 from household members) were successfully sequenced for STs and alleles analysis. Nineteen samples with low quality sequences were excluded from further analysis. Among the isolates from family members, four of them were from individuals (HM50C, HM50D, HM59B and HM59C) living with a Blastocystis-negative daycare child (Appendix 1). All the 57 isolates had single ST infections and none evidence of mixed infections was found. The overall prevalence of Blastocystis subtypes was as follows: ST1 (31/57; 54.4\%), ST2 (4/57; $7.0 \%)$, ST3 (19/57; 33.3\%) and ST7 (3/57; 5.3\%). Among the isolates from children, ST1 $(57.9 \%)$ was the most common, followed by ST3 (26.3\%), ST2 (10.5\%) and ST7 (5.3\%) (Table 1; Supplementary Appendix 1). Four samples from staff members were found positive for two STs: ST1 $(n=2)$ and ST3 $(n=2)$. In addition, 15 isolates retrieved

Table 1

Prevalence and subtype distribution of Blastocystis isolates infecting daycare children, household members and care workers.

\begin{tabular}{|c|c|c|c|c|c|c|c|}
\hline \multirow[t]{2}{*}{ Samples (n) } & \multirow[t]{2}{*}{ No. } & \multirow[t]{2}{*}{ No. PCR Positive } & \multicolumn{4}{|l|}{ Subtype (\%) } & \multirow[t]{2}{*}{ Total } \\
\hline & & & ST1 & ST2 & ST3 & ST7 & \\
\hline Children & 123 & 50 & $23(57.9)$ & $04(10.5 \%)$ & $10(26.3)$ & $02(5.7 \%)$ & 38 \\
\hline Household members & 44 & 22 & $07(46.7)$ & - & 07 (46.7) & $01(6.7)$ & 15 \\
\hline Staff members & 14 & 04 & $02(50.0)$ & - & $02(50.0)$ & - & 04 \\
\hline Total & 181 & 76 & $31(54.4 \%)$ & $04(7.0)$ & $19(33.3)$ & $03(5.3)$ & 57 \\
\hline
\end{tabular}

Blatocystis subtypes distribution between daycare children, workers and household members were tested for significance by the adjusted Wald test $(P<0.0001)$. 
from household members (14 adults and one children) were distributed among the following STs: ST1 (46.7\%), ST3 (46.7\%) and, ST7 (6.7\%) (Table 1, Appendix 1). In the surveyed population, prevalence of Blastocystis ST1 and ST3 infections was significantly higher among daycare children and household members $(p<0.0001)$. However, in the former group, ST3 was significantly less common than ST1 $(p<0.0001)$. Moreover, prevalence of ST2 and ST7 was higher among children $(p<0.0001)$.

Phylogenetic analysis showed clear distribution of isolates into four distinct STS (Supplementary Fig. S1). The comparison of sequences classified as ST1, ST2, ST3 showed 92-99\%, 99\% and 98-99\% identity, respectively, with reference sequences previously described. Among the three ST7 sequences (Supplementary Fig. S1), two of them, namely a daycare children isolate (DC44) and its respective household isolate (HM44B) showed significant identity to a human isolate (100\%) identified in Brazil (GenBank KP734167.1) and to a chicken isolate (97\%) identified in Malaysia (GenBank KX234592.1). The third ST7 isolate (DC33) retrieved from another daycare child was less closely related to the same human and chicken isolates, showing $93 \%$ and $91 \%$ of identity, respectively. However in this particular case, the isolates recovered from her household members were assigned as ST1 (HM33C and HM33D).

At the intra-ST level, allele assignment was obtained for all sequences. For ST1 isolates, almost $94 \%(n=32)$ belonged to allele 4 and only two were classified as alleles 78 and 80, respectively. For ST2 two alleles were detected (alleles 11 and 12), for ST3 three alleles (34, 36 and 37) and for ST7 two alleles (106 and 137). Among the ST3 isolates $(\mathrm{n}=18)$, the second most prevalent subtype, allele 34 was identified in $55.6 \%$ of the sequences (Supplementary Appendix 1).

\section{Discussion}

Nowadays, molecular surveys have allowed a more specific and sensitive detection of Blastocystis infection in a wide range of human population groups resulting in a clearer understanding of its epidemiology. In the current study, we investigated the prevalence and distribution of Blastocystis subtypes in a daycare center serving a low-income community. Here, results revealed a great prevalence of Blastocystis, as, from the 181 human samples tested by PCR, 76 (41.9\%) were positive. Among daycare children, Blastocystis was detected in $40.7 \%$ of the subjects and infection was also observed in $28.6 \%$ and $50 \%$ of staff and household members, respectively. These results are consistent with recent surveys in South America, including in Brazil, focused on several cohorts of population such as children (Dib et al., 2015; Rebolla et al., 2016; Ramirez et al., 2017; Sánchez et al., 2017), population in slums (Faria et al., 2017; Gil et al., 2013), indigenous communities (Malheiros et al., 2011; Sánchez et al., 2017), rural communities (Incani et al., 2017; Macchioni et al., 2016; Machicado et al., 2012), fishing village dwellers (David et al., 2015) and individuals visiting hospitals (Casero et al., 2015; Melo et al., 2017). In all these groups, Blastocystis has been often diagnosed with infection rates of $<15 \%$ to as high as $50 \%$. However, children can be more sensitive to infection with a very high overall prevalence of $90 \%$ detecting by PCR as previously observed in cohorts of children living in different geographical regions of Colombia (Ramirez et al., 2017). The wide range of prevalence rates reported in different studies may be due to reasons as, for example, survey population, geographic location, and diagnostic methods. Interestingly, on a survey carried out for over 20 years at the daycare center of the current study, 39.1\% of infection was shown by microscopic examination after iodine and iron-hematoxylin staining procedures (Guimarães and Sogayar, 1993). In fact, this prevalence would have been higher if PCR had been employed as recently demonstrated in molecular surveys in Brazil (David et al., 2015) and Colombia (Sánchez et al., 2017). It is important to stress that, when the same 123 fecal samples from children were assessed by microscopic examination in a previous survey (Oliveira-Arbex et al., 2016),
Blastocystis infection showed a lower frequency (8.9\%) than $40.7 \%$ estimated by PCR in the current study. Moreover, infection among staff and household members that had not previously been detected by microscopy could be observed by PCR in $28.6 \%$ and $50 \%$, respectively.

With regards to the possibility of isolating identical STs from humans and their in-contact animals, Blastocystis was investigated in 20 stool samples collected from dogs owned by the children's families, however, none was found positive by PCR. Since the ubiquity of Blastocystis and the fact that infection has been reported in animals with proximity to humans, detection in canine fecal samples would be expected. However, as properly addressed by Cian et al. (2017) in a recent publication proposed to identify potential animal reservoirs of human infection in France, in a great part of surveys to assess infection in nonhuman hosts, dogs have been either found to be negative for (Abe et al., 2002; David et al., 2015; Spanakos et al., 2011) or infected by the parasite with a low or moderate prevalence ranging between $1.3 \%$ and 14.5\% (Belleza et al., 2015; La Sala et al., 2015; Osman et al., 2015; Ruaux and Stang, 2014; Wang et al., 2013). Furthermore, these authors highlight the findings of two investigations in which the prevalence of Blastocystis was significantly higher in stray dogs living in low-income areas deprived of hygiene and sanitation conditions (Ramirez et al., 2014; Wang et al., 2013). Similarly, our study was conducted in a lowincome area. However, most of the community population has access to sanitation facilities, preventing the environmental contamination by human fecal material and reducing the risk for dogs to acquire the infection.

Current studies on Blastocystis genetic diversity have improved the possibility to achieve better understanding of infection dynamics. In the present study, of the 76 PCR-positive samples, 25\% (19) were not successfully sequenced and subtyped. Although the reasons for this are unclear, this failure may be a consequence of the poor concentration of DNA template, especially in low-density infections. This is a relevant condition that can lead to no PCR product or yielded poor amplification. Studies analyzing infection intensity by microscopy examination have shown higher percentages of stool samples with few to moderate parasites than those with parasite abundance (Leder et al., 2005; Özyurt et al., 2008). Recently, Melo et al. (2017) observed that 13 of 60 Blastocystis positive samples by microscopic examination failed to produce PCR products and interestingly, five of which were reported as rare, four as few to moderate and four as suggestive forms by parasitological analyses. These authors also reported that of 47 PCR positive samples sequenced, seven sequences were excluded from the analysis, as they presented low-quality possibly due to poor amplification of DNA. In a recent molecular-based survey conducted among Nigerian children (Poulsen et al., 2016), positive samples by real-time PCR were subtyped by both barcoding region sequencing (Scicluna et al., 2006) and sequence-tagged-site PCR (Stensvold, 2013b). Some samples contained so little Blastocystis DNA (evidenced by high qPCR cycle threshold values) was unsuccessful subtyped when the former method was used. Such is another factor that could be raised in the present study since samples were subtyped by the method proposed by Scicluna et al. (2006).

Regarding the distribution of subtypes, ST1 (56.1\%), ST2 (7.0\%), ST3 (31.6\%) and ST7 (5.3\%) were identified among 57 human isolates successfully genotyped. All these isolates had single ST infections and thus, none mixed infections could be observed. In the present study, the distribution of Blastocystis subtypes was broadly similar to that reported in previous surveys worldwide in which the majority of human infections have been assigned to STs 1, 2 and 3. Up to now, nine subtypes have been found in humans. However, $90-95 \%$ of human infections belonged to one of ST1-ST4 subtypes, with a predominance of ST3 (Stensvold and Clark, 2016). Globally, ST3 predominance has been observed in different geographic areas and populations, including in South America (David et al., 2015; Melo et al., 2017; Ramirez et al., 2014, 2016; Sánchez et al., 2017). Otherwise, our results showed the predominance of ST1 $(56.1 \%)$ and this observation concurs to findings 
of some particular investigations in Africa (Forsell et al., 2016), Asia (Sanpool et al., 2017; Thathaisong et al., 2013; Yoshikawa et al., 2016b) and in some countries in Latin America as Brazil, Colombia and Mexico (Malheiros et al., 2011; Ramirez et al., 2014; Villegas-Gómez et al., 2016). Even though ST3 was not the most prevalent subtype in the sampled population, its prevalence (31.6\%) was relevant.

In our study, ST1 and ST3 were found infecting daycare children, staff and household members, however, the predominance of these subtypes among children and their families were found to be significantly different. Especially among children, the prevalence of ST1 was significantly higher than ST3. Despite this evidence, it is still difficult to establish a specific epidemiological relationship with these subtypes because the sample size was small. However, in a community with access to basic sanitary facilities, high prevalence of two subtypes commonly found in human infections could suggest person-to-person transmission as a usual route within families and institution. In these groups, poor personal hygiene and lack of regular hand washing habit favor parasite transmission.

About ST2, it was observed only in children (four samples). ST2 is one of the common subtypes in human infections, but interestingly in some studies it has been detected at lower ratios than ST1 and ST3 or it is absent in a given population (Pandey et al., 2015; Villegas-Gómez et al., 2016). Apart from this, it is worth noting that ST4 was absent in the present study which reinforces the fact that this subtype is rarely detected in South America's human populations, as previously shown (David et al., 2015; Malheiros et al., 2011; Melo et al., 2017). To this date, ST4 has shown a restricted distribution, being rare or absent in South America, North Africa, and the Middle East, however, this subtype is the second most common subtype after ST3 in Europe (Stensvold and Clark, 2016). Lately, its presence has been sporadically reported in humans in Colombia, but always at low frequencies (Ramirez et al., 2016, 2017; Sánchez et al., 2017). It is not clear why ST4 is uncommon or absent outside European countries, but as highlighted by Clark et al. (2013), the genetic homogeneity intra-ST4 in comparison with subtypes 1,2 and 3 has led to speculatin that ST4 entered the human population fairly recently than the other subtypes.

Another point that deserves mentioning is the presence of human infection with ST7. Subtypes 6 and 7 are usually found in birds and they have been isolated from humans only occasionally (Clark et al., 2013; Stensvold et al., 2009). Very little data is currently available on human infections with ST6 and ST7. However, their presence has been revealed in Africa (Forsell et al., 2016), Asia (Palasuwan et al., 2016; Sanpool et al., 2017; Thathaisong et al., 2013; Yoshikawa et al., 2016b) and more recently in South America (David et al., 2015; Melo et al., 2017; Ramirez et al., 2016, 2017; Sánchez et al., 2017).

In the present study, ST7 isolates were found infecting only two daycare children aged 1-year and 5-year old and also a 6-year-old sister of the youngest child. Curiously, during an interview, parents/guardians of these children informed that they raised free-living chicken in the households to provide food for families. They also reported that chickens were kept close to children since these birds were kept in backyards. In this context, children are more likely to become infected during outdoor activities in an environment contaminated by chicken feces. This situation reinforces the statement that birds, especially poultry, can be considered as potential reservoirs for human infections. Moreover, previous studies have reported that domestic birds reared in different areas are frequently infected with Blastocystis (Alfellani et al., 2013b; Lee and Stenzel, 1999; Yoshikawa et al., 2016b). The fact that stool samples from chicken were not analyzed during the survey may be a limitation to conclude that zoonotic transmission was present in this scenario. Even so, this possibility should not be ruled out, since nucleotide sequences retrieved from two children living in the same household showed high identity with each other (100\%) and also when compared to a chicken isolate identified in Malaysia (97\%). However, the third ST7 isolate was less closely related to the same chicken reference sequence $(91 \%)$.
Blastocystis infections have been detected in both individuals with gastrointestinal symptoms and healthy ones. Albeit studies have focused on the occurrence of Blastocystis subtypes between asymptomatic and symptomatic individuals, so far, no particular subtype has been yet linked consistently to disease (Stensvold and Clark, 2016). In the present study, considering that all children were characterized as asymptomatic, attempts to verify a likely association between subtypes and clinical manifestations were not considered. Nevertheless, asymptomatic carriers are common in endemic areas and the epidemiological relevance of this group should not be underestimated. Particularly in establishments for childcare, asymptomatic young children without personal hygiene living in close contact may serve as healthy carriers of infectious agents, including Blastocystis, disseminating them to other children as well to the surrounding community.

As for intra-subtype variation, $18 \mathrm{~S}$ alleles analysis of each ST revealed a total of 10 different alleles within the studied population as follows: alleles 4, 78 and 80 for ST1; alleles 11 and 12 for ST2; alleles 34, 36, 37 for ST3; and alleles 106 and 137 for ST7. Among these variants, allele 4 from ST1 was the most frequent, followed by allele 34 from ST3. Similarly to subtypes frequencies, the occurrence of alleles within a given population is variable (Scanlan et al., 2014). However, these two alleles have been reported to be common variants related to human infections elsewhere, including in South America (David et al., 2015; Ramirez et al., 2016, 2017; Poulsen et al., 2016; Sánchez et al., 2017). Studies comparing subtypes and their respective alleles isolated from certain host groups have enabled a better understanding about Blastocystis transmission (Andersen and Stensvold, 2016). In that way, in the present study, some findings deserve to be highlighted, such as the higher frequency $(50.9 \%)$ of allele 4 associated with infections in the studied population, especially among daycare children (57.7\%). Such interesting finding lead us to rise that this variant is closely related to person-to-person transmission and it is most likely play a role in the dissemination of Blastocystis in this particular environment. Not only among the daycare children, but allele 1 was also found in daycare workers (two isolates) and in household members (five isolates). In support of our results, Blastocystis ST1/variant 1 was reported as the most predominant among households of an orphanage for girls in Thailand, suggesting that the infection occurred within the community and probably by person-to-person transmission (Thathaisong et al., 2013).

Although the surveyed population and the number of characterized isolates were relatively small, data assembled herein provided interesting insights on epidemiology and genetic diversity of Blastocystis infecting subjects of a community of lower socioeconomic status where conditions are favorable and ensure its transmission. Whether pathogenic or not, data on Blastocystis prevalence and subtypes distribution reported in different parts of the world are necessary to reinforce its relevance since to date this parasite is frequently dismissed by many clinicians as an organism of no importance (Clark et al., 2013).

\section{Conflict of interest}

The authors declare no conflict of interest.

Supplementary data to this article can be found online at https:// doi.org/10.1016/j.meegid.2017.11.005.

\section{Acknowledgments}

This work was funded by the Fundação de Amparo à Pesquisa do Estado de São Paulo (FAPESP) grants (2011/52100-3). We are very grateful to all individuals for their participation in the study.

\section{References}

Abe, N., Nagoshi, M., Takami, K., Sawano, Y., Yoshikawa, H., 2002. A survey of Blastocystis sp. in livestock, pets and zoo animals in Japan. Vet. Parasitol. 106, 
203-212.

AbuOdeh, R., Ezzedine, S., Samie, A., Stensvold, C.R., ElBakri, A., 2016. Prevalence and subtype distribution of Blastocystis in healthy individuals in Sharjah, United Arab Emirates. Infect. Genet. Evol. 37, 158-162.

Alfellani, M.A., Stensvold, C.R., Vidal-Lapiedra, A., Onuoha, E.S., Fagbenro-Beyioku, A.F., Clark, C.G., 2013a. Variable geographic distribution of Blastocystis subtypes and its potential implications. Acta Trop. 126, 11-18.

Alfellani, M.A., Taner-Mulla, D., Jacob, A.S., Imeede, C.A., Yoshikawa, H., Stensvold, C.R., Clark, C.G., 2013b. Genetic diversity of Blastocystis in livestock and zoo animals. Protist 164, 497-509.

Andersen, L.O., Stensvold, C.R., 2016. Blastocystis in health and disease: are we moving from a clinical to a public health perspective? J. Clin. Microbiol. 54, 524-528.

Belleza, M.L., Cadacio, J.L., Borja, M.P., Solon, J.A., Padilla, M.A., Tongol-Rivera, P.N., Rivera, W.L., 2015. Epidemiologic study of Blastocystis infection in an urban community in the Philippines. J. Environ. Public Health 894297.

Casero, R., Mongi, F., Sánchez, A., Ramírez, J.D., 2015. Blastocystis and urticaria: examination of subtypes and morphotypes in an unusual clinical manifestation. Acta Trop. 126, 12-16.

Cian, A., El Safadi, D., Osman, M., Moriniere, R., Gantois, N., Benamrouz-Vanneste, S., et al., 2017. Molecular epidemiology of Blastocystis sp. in various animal groups from two French zoos and evaluation of potential zoonotic risk. PLoS One 12 (1), e0169659.

Clark, C.G., van der Giezen, M., Alfellani, M.A., Stensvold, C.R., 2013. Recent developments in Blastocystis research. Adv. Parasitol. 82, 1-32.

David, E.B., Guimarães, S., de Oliveira, A.P., Oliveira-Sequeira, T.C.G., Nogueira, G.B., Nardi, A.R.M., Ribolla, P.E., Franco, R.M.B., Branco, N., Tosini, F., Bella, A., Pozio, E., Cacciò, S.M., 2015. Molecular characterization of intestinal protozoa in two poor communities in the state of Sao Paulo, Brazil. Parasites Vect. 8, 103.

Dib, J.R., Fernández-Zenoff, M.V., Oquilla, J., Lazarte, S., González, S.N., 2015. Prevalence of intestinal parasitic infection among children from a shanty town in Tucuman, Argentina. Trop. Biomed. 32, 210-215.

Dogan, N., Aydin, M., Tuzemen, N.U., Dinleyici, E.C., Oguz, I., Dogruman-Al, F., 2017 Subtype distribution of Blastocystis spp. isolated from children in Eskisehir, Turkey. Parasitol. Int. 66, 948-951.

El Safadi, D., Gaayeb, L., Meloni, D., Cian, A., Poirier, P., Wawrzyniak, I., Delbac, F., Dabboussi, F., Delhaes, L., Seck, M., Hamze, M., Riveau, G., Viscogliosi, E., 2014. Children of Senegal River Basin show the highest prevalence of Blastocystis sp. ever observed worldwide. BMC Infect. Dis. 14, 164.

Faria, C.P., Zanini, G.M., Dias, G.S., da Silva, S., de Freitas, M.B., Almendra, R., Santana, P., Sousa, M.D., 2017. Geospatial distribution of intestinal parasitic infections in Rio de Janeiro (Brazil) and its association with social determinants. PLoS Negl. Trop. Dis. 11, e0005445.

Forsell, J., Granlund, M., Samuelsson, L., Koskiniemi, S., Edebro, H., Evengård, B., 2016. High occurrence of Blastocystis sp. subtypes 1-3 and Giardia intestinalis assemblage B among patients in Zanzibar, Tanzania. Parasit. Vectors 29, 370.

Gil, F.F., Busatti, H.G., Cruz, V.L., Santos, J.F., Gomes, M.A., 2013. High prevalence of enteroparasitosis in urban slums of Belo Horizonte-Brazil. Presence of enteroparasites as a risk factor in the family group. Pathog. Glob. Health 107, 320-324.

Guimarães, S., Sogayar, M.I., 1993. Blastocystis hominis: occurrence in children and staff members of municipal day-care centers from Botucatu, São Paulo State, Brazil. Mem. Inst. Oswaldo Cruz 88, 427-429.

Incani, R.N., Ferrer, E., Hoek, D., Ramak, R., Roelfsema, J., Mughini-Gras, L., Kortbeek, T., Pinelli, E., 2017. Diagnosis of intestinal parasites in a rural community of Venezuela: advantages and disadvantages of using microscopy or RT-PCR. Acta Trop. 167, 64-70.

La Sala, L.F., Leiboff, A., Burgos, J.M., Costamagna, S.R., 2015. Spatial distribution of canine zoonotic enteroparasites in Bahia Blanca, Argentina. Rev. Argent. Microbiol. 47, 17-24.

Larkin, M.A., Blackshields, G., Brown, N.P., Chenna, R., McGettigan, P.A., McWilliam, H., Valentin, F., Wallace, I.M., Wilm, A., Lopez, R., Thompson, J.D., Gibson, T.J., Higgins, D., 2007. Clustal W and clustal X version 2.0. Bioinformatics 23, 2947-2948.

Leder, K., Hellard, M., Sinclair, M.I., Fairley, C.K., Wolfe, R., 2005. No correlation between clinical symptoms and Blastocystis hominis in immunocompetent individuals. J. Gastroenterol. Hepatol. 20, 1390-1394.

Lee, M.G., Stenzel, D.J., 1999. A survey of Blastocystis in domestic chickens. Parasitol. Res. 85, 109-117.

Machicado, J.D., Marcos, L.A., Tello, R., Canales, M., Terashima, A., Gotuzzo, E., 2012. Diagnosis of soil-transmitted helminthiasis in an Amazonic community of Peru using multiplediagnostic techniques. Trans. R. Soc. Trop. Med. Hyg. 106, 333-339.

Macchioni, F., Segundo, H., Totino, V., Gabrielli, S., Rojas, P., Roselli, M., Paredes, G.A., Masana, M., Bartoloni, A., Cancrini, G., 2016. Intestinal parasitic infections and associated epidemiological drivers in two rural communities of the Bolivian Chaco. J. Infect. Dev. Ctries. 10, 1012-1019.

Malheiros, A.F., Stensvold, C.R., Clark, C.G., Braga, G.B., Shaw, J.J., 2011. Short report: molecular characterization of Blastocystis obtained from members of the indigenous Tapirape ethnic group from the Brazilian Amazon region, Brazil. Am. J. Trop. Med. Hyg. 85, 1050-1053.

Melo, G.B., Paula, F.M., Malta, F.M., Maruta, C.W., Criado, P., Castilho, V.L.P., Gonçalves, E.M.N., Espirito Santo, M.C., Gryschek, R.C.B., 2017. Identification of Blastocystis subtypes in clinical stool samples from Sao Paulo City, Brazil. Parasitol. Open. 3. http://dx.doi.org/10.1017/pao.2017.3.

Nithyamathi, K., Chandramathi, S., Kumar, S., 2016. Predominance of Blastocystis sp. infection among school children in Peninsular Malaysia. PLoS One 11, e0136709.
Oliveira-Arbex, A.P., David, E.B., Oliveira-Sequeira, T.C., Bittencourt, G.N., Guimarães, S., 2016. Genotyping of Giardia duodenalis isolates in asymptomatic children attending daycare center: evidence of high risk for anthroponotic transmission. Epidemiol. Infect. 144, 1418-1428.

Osman, M., Bories, J., El Safadi, D., Poirel, M.T., Gantois, N., Benamrouz-Vanneste, S., Delhaes, L., Hugonnard, M., Certad, G., Zenner, L., Viscogliosi, E., 2015. Prevalence and genetic diversity of the intestinal parasites Blastocystis sp. and Cryptosporidium spp. in household dogs in France and evaluation of zoonotic transmission risk. Vet. Parasitol. 214, 167-170.

Özyurt, M., Kurt, O., Mølbak, K., Nielsen, H.V., Haznedaroglu, T., Stensvold, C.R., 2008 Molecular epidemiology of Blastocystis infections in Turkey. Parasitol. Int. 57, 300-306.

Palasuwan, A., Palasuwan, D., Mahittikorn, A., Chiabchalard, R., Combes, V., Popruk, S. 2016. Subtype distribution of Blastocystis in communities along the Chao Phraya River, Thailand. Korean J. Parasitol. 54, 455-460.

Pandey, P.K., Verma, P., Marathe, N., Shetty, S., Bavdekar, A., Patole, M.S., Stensvold, C.R., Shouche, Y.S., 2015. Prevalence and subtype analysis of Blastocystis in healthy Indian individuals. Infect. Genet. Evol. 31, 296-299.

Poirier, P., Wawrzyniak, I., Vivares, C.P., Delbac, F., El Alaoui, H., 2012. New insights into Blastocystis spp.: a potential link with irritable bowel syndrome. PLoS Pathog. 8, e1002545.

Poulsen, C.S., Efunshile, A.M., Nelson, J.A., Stensvold, C.R., 2016. Epidemiological aspects of Blastocystis colonization in children in Ilero, Nigeria. Am. J. Trop. Med. Hyg. 95, 175-179.

Ramirez, J.D., Sanchez, L.V., Bautista, D.C., Corredor, A.F., Florez, A.C., Stensvold, C.R., 2014. Blastocystis subtypes detected in humans and animals from Colombia. Infect. Genet. Evol. 22, 223-228.

Ramirez, J.D., Sánchez, A., Hernández, C., Flórez, C., Bernal, M.C., Giraldo, J.C., Reyes, P., López, M.C., García, L., Cooper, P.J., Vicuña, Y., Mongi, F., Casero, R.D., 2016. Geographic distribution of human Blastocystis subtypes in South America. Infect. Genet. Evol. 41, 32-35.

Ramirez, J.D., Flórez, C., Olivera, M., Bernal, M.C., Giraldo, J.C., 2017. Blastocystis subtyping and its association with intestinal parasites in children from different geographical regions of Colombia. PLoS One 12, e0172586.

Rebolla, M.F., Silva, E.M., Gomes, J.F., Falcão, A.X., Rebolla, M.V., Franco, R.M., 2016. High prevalence of Blastocystis spp. infection in children and staff members attending public urban schools in São Paulo State, Brazil. Rev. Inst. Med. Trop. Sao Paulo $58,31$.

Ruaux, C.G., Stang, B.V., 2014. Prevalence of Blastocystis in shelter-resident and clientowned companion animals in the US Pacific Northwest. PLoS One 9, e107496.

Sánchez, A., Munoz, M., Gómez, N., Tabares, J., Segura, L., Salazar, Á., Restrepo, C., Ruíz M., Reyes, P., Qian, Y., Xiao, L., López, M.C., Ramírez, J.D., 2017. Molecular epidemiology of Giardia, Blastocystis and Cryptosporidium among indigenous children from the Colombian Amazon Basin. Front. Microbiol. 8, 248.

Sanpool, O., Laymanivong, S., Thanchomnang, T., Rodpai, R., Sadaow, L., Phosuk, I., Maleewong, W., Intapan, P.M., 2017. Subtype identification of human Blastocystis spp. isolated from Lao People's Democratic Republic. Acta Trop. 68, 37-40.

Scanlan, P.D., Stensvold, C.R., Rajilić-Stojanović, M., Heilig, H.G., De Vos, W.M., O'Toole, P.W., Cotter, P.D., 2014. The microbial eukaryote Blastocystis is a prevalent and diverse member of the healthy human gut microbiota. FEMS Microbiol. Ecol. 90, 326-330.

Scicluna, S., Tawari, B., Clark, C., 2006. DNA barcoding of Blastocystis. Protist 157, 77-85.

Spanakos, G., Papadogiannakis, E., Kontos, V., Menounos, P., Velonakis, E., Koutis, C. Vakalis, N.C., 2011. Molecular screening for Blastocystis sp. in canine faecal samples in Greece. J. Hell. Vet. Med. Soc. 62, 216-220.

Stensvold, C.R., 2013a. Blastocystis: genetic diversity and molecular methods for diagnosis and epidemiology. Trop. Parasitol. 31, 26-34.

Stensvold, C.R., 2013b. Comparison of sequencing (barcode region) and sequence-taggedsite PCR for Blastocystis subtyping. J. Clin. Microbiol. 51, 190-194.

Stensvold, C.R., Clark, C.G., 2016. Current status of Blastocystis: a personal view. Parasitol. Int. 65, 763-771.

Stensvold, C.R., Lewis, H.C., Hammerum, A.M., Porsbo, L.J., Nielsen, S.S., Olsen, K.E. Arendrup, M.C., Nielsen, H.V., Molbak, K., 2009. Blastocystis: unravelling potential risk factors and clinical significance of a common but neglected parasite. Epidemiol. Infect. 137, 1655-1663.

Tan, K.S., 2008. New insights on classification, identification, and clinical relevance of Blastocystis spp. Clin. Microbiol. Rev. 21, 639-665.

Thathaisong, U., Siripattanapipong, S., Mungthin, M., Pipatsatitpong, D., Tan-ariya, P., Naaglor, T., Leelayoova, S., 2013. Identification of Blastocystis subtype 1 variants in the Home for Girls, Bangkok, Thailand. Am. J. Trop. Med. Hyg. 88, 352-358.

Villegas-Gómez, I., Martínez-Hernández, F., Urrea-Quezada, A., González-Díaz, M., Durazo, M., Hernández, J., Orozco-Mosqueda, G.E., Villalobos, G., Maravilla, P., Valenzuela, O., 2016. Comparison of the genetic variability of Blastocystis subtypes between human carriers from two contrasting climatic regions of México. Infect. Genet. Evol. 44, 334-340.

Wang, W., Cuttell, L., Bielefeldt-Ohmann, H., Inpankaew, T., Owen, H., Traub, R.J., 2013. Diversity of Blastocystis subtypes in dogs in different geographical settings. Parasit. Vectors 6, 215.

Yoshikawa, H., Koyama, Y., Tsuchiya, E., Takami, K., 2016a. Blastocystis phylogeny among various isolates from humans to insects. Parasitol. Int. 65, 750-759.

Yoshikawa, H., Tokoro, M., Nagamoto, T., Arayama, S., Asih, P.B., Rozi, I.E., Syafruddin, D., 2016b. Molecular survey of Blastocystis sp. from humans and associated animals in an Indonesian community with poor hygiene. Parasitol. Int. 65, 780-784. 